\title{
Impact of the COVID-19 Pandemic on Surgical Colorectal Cancer Care in the Netherlands: a Multicenter Retrospective Cohort Study
}

\author{
Mando Filipe $^{1} \cdot$ Ellen de Bock ${ }^{1} \cdot$ Ritch Geitenbeek $^{2} \cdot$ Djamila Boerma $^{3} \cdot$ Apollo Pronk $^{4} \cdot$ Joost Heikens $^{3,5} \cdot$ Milan Richir $^{1}$
}

Received: 20 November 2020 / Accepted: 18 January 2021 / Published online: 23 February 2021

(C) 2021 The Author(s)

Keywords COVID-19 $\cdot$ Surgery $\cdot$ Pandemic $\cdot$ Complications $\cdot$ Colorectal cancer $\cdot$ Multicenter

\section{Introduction}

Coronavirus disease 2019 (COVID-19) poses a challenge to routine healthcare practice.

The suspension of the national screening program combined with fewer referrals through primary care (the general practitioner) has led to fewer colorectal cancer diagnoses in the Netherlands. ${ }^{1}$ However, the consequences of the COVID19 pandemic for colorectal cancer surgical care are unknown. Therefore, the aim of this study is to determine the impact of the COVID-19 pandemic on the surgical management of colorectal cancer.

\section{Methods}

This retrospective cohort study included all patients with colorectal cancer who were operated on between March 9 and June 30, 2020, in four hospitals across the Netherlands. One of these was an academic hospital, and the other three were district general hospitals. The primary outcome was the number of colorectal cancer resections performed. Secondary endpoints were the number and risk factors of postoperative complications in patients undergoing colorectal cancer surgery.

Mando Filipe and Ellen de Bock contributed equally to this work.

Mando Filipe

m.d.filipe-2@umcutrecht.nl

Milan Richir

m.c.richir@umcutrecht.nl

1 Department of Surgery, Cancer Centre, University Medical Centre Utrecht, Utrecht, The Netherlands
Multivariate logistic regression analysis was performed to ascertain the risk of developing a complication (infections, bleeding, ICU admission, and/or anastomotic leakage) in patients who underwent colorectal cancer surgery after correcting for possible confounders. Odds ratio (OR) with 95\% confidence intervals (CI) were used to quantify the risk of postoperative complications. Two-sided $\mathrm{P}$ values below 0.05 were considered statistically significant.

\section{Results}

One hundred sixty-two patients underwent colorectal resectional surgery for malignant pathology. There were 88 lower staged (stages $0-$ II) and 74 higher staged (stages III-IV) colorectal cancers. Of the 162 patients, 21 had been tested for COVID-19 (13.0\%), two of whom tested positive (9.5\%). Postoperative complications occurred in 46 (28.4\%) patients. An overall reduction in the overall numbers of colorectal resections occurred over the time of the study (Fig. 1a). After week 22, no colorectal cancer resections were performed for patients diagnosed through the national screening program (Fig. 1b). Furthermore, an overall decrease was observed in the number of procedures performed for patients with colorectal cancer who were referred through primary care. The number of colorectal cancer procedures performed for patients diagnosed with low-stage tumors gradually declined during the

2 Department of Surgery, Meander Medical Centre, Amersfoort, The Netherlands

3 Department of Surgery, St. Antonius Hospital, Nieuwegein, The Netherlands

4 Department of Surgery, Diakonessenhuis, Utrecht, The Netherlands

5 Department of Surgery, Rivierenland Hospital, Tiel, The Netherlands 
course of the study period (Fig. 1c), while the high-stage tumors remained stable. During the study period, the number and risk (OR $1.03 ; 95 \%$ CI $0.94-1.12 ; \mathrm{P}=0.573$ ) of postoperative complications remained stable (Fig. 1d and Table 1).

\section{Discussion}

The current study showed a decline of colorectal surgical procedures during the study period. This appears to be a result of the suspension of the national colorectal screening program as well as a reduction in the number of referrals from primary care. The result of this has been a decrease in the apparent incidence of lower staged tumors. This is unsurprising, since colorectal cancers diagnosed at the national screening program are detected at an early stage compared to patients who present with colorectal-related symptoms. ${ }^{1,2}$ The national screening program restarted on June 1, 2020. However, this has not (yet) led to an increase in colorectal surgical procedures performed. Possible reasons could be that the screening program has not yet fully started (70\% of the invitation rate). Additionally, the colorectal cancer screening program consists of a fecal immunochemical test (FIT) in the first instance. Only if FIT is positive will an endoscopy be offered. ${ }^{3}$

This study also showed that the number of patients presenting with symptomatic colorectal cancer (non-screened patients) decreased during the COVID-19 period which could be a representation of the decreased referrals by primary care.

Since the ongoing COVID-19 pandemic puts an enormous strain on healthcare systems worldwide, this study showed that, if necessary, suspension of the national screening program is acceptable as long as explicit attention is paid to the continuation primary care referrals. a

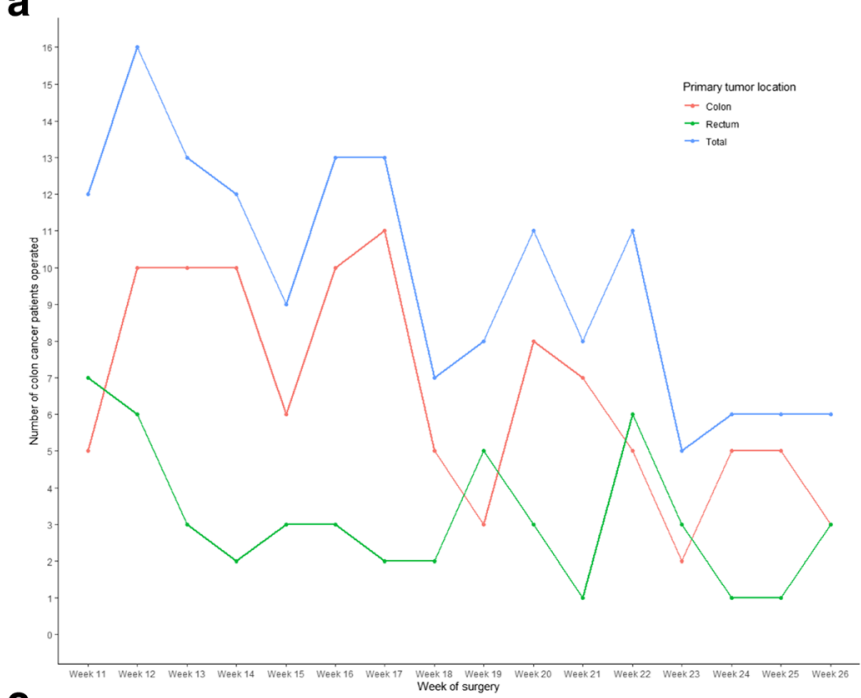

C

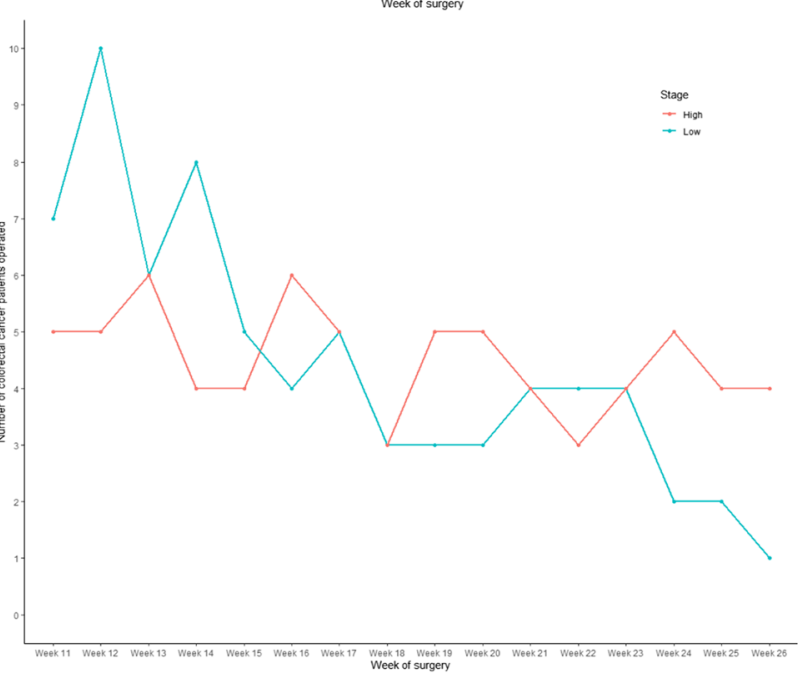

Fig. 1 a-d. a Top left = number of colorectal cancer surgical procedures stratified by colon and rectum. b Top right $=$ number of patients with colorectal cancer who had undergone surgical procedures from March 9 to June 30,2020 , with referral from national screening program or general b
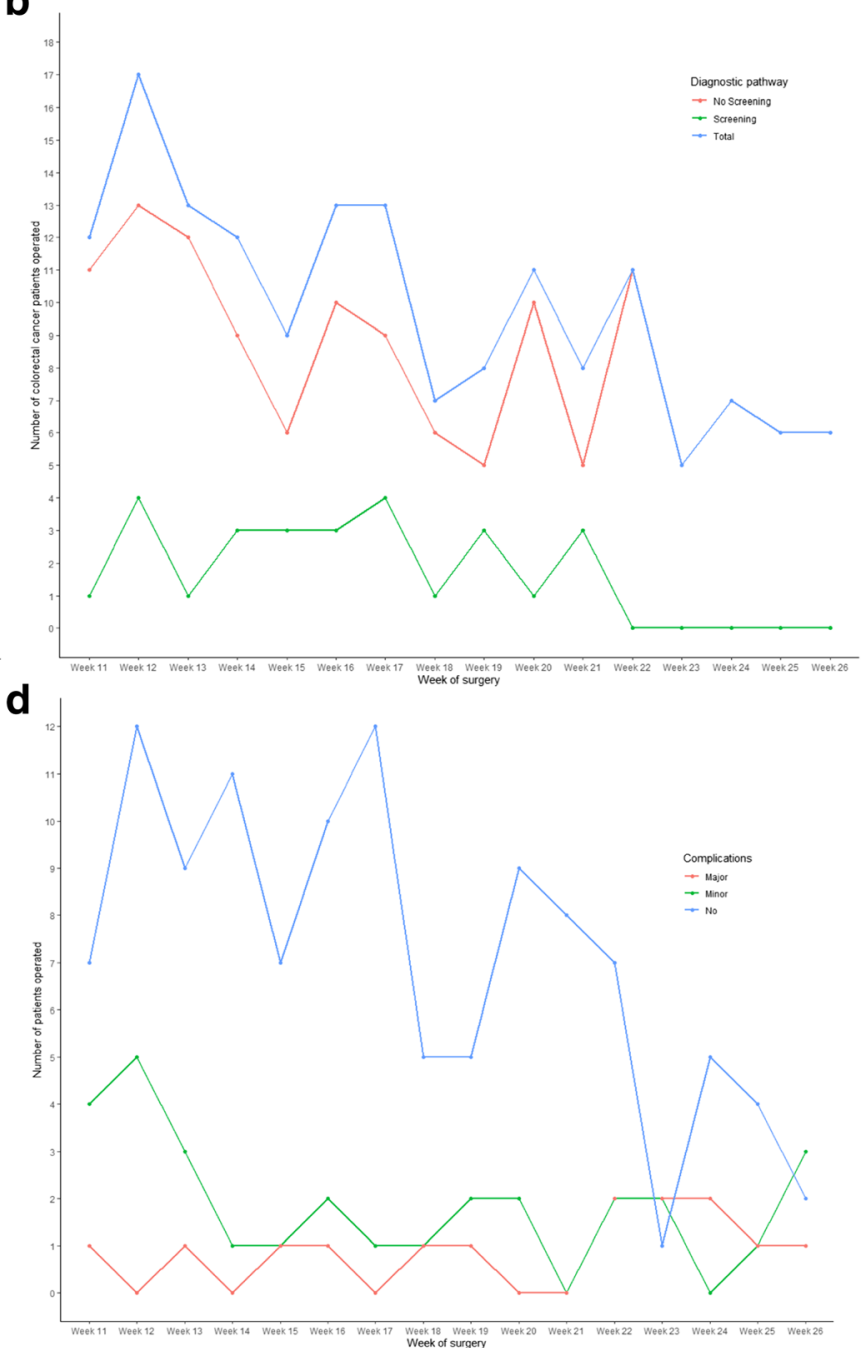

practitioner. $\mathbf{c}$ Bottom right $=$ number of colorectal cancer surgical procedures presented by tumor stage. Low $=$ stage Tis, stage I and stage II. High = stage III and stage IV . d Bottom left $=$ number of colorectal cancer surgical procedures presented by type of complications 
Table 1 Multivariate analysis for the risk of complications

\begin{tabular}{llllll}
\hline & Estimate & OR $(\mathrm{CI})$ & Standard error & $Z$ value & $P$ value \\
\hline Age in years & 0.017 & $1.02(0.98-1.05)$ & 0.019 & 0.917 & 0.359 \\
Sex & & & & \\
Sex, female & NA & 1.00 (reference) & NA & NA & NA \\
Sex, male & 0.858 & $2.36(0.93-5.98)$ & 0.474 & 1.809 & 0.070 \\
BMI & 0.000 & $1.00(0.91-1.10)$ & 0.048 & -0.01 & 0.992 \\
Number of comorbidities & -0.086 & $0.92(0.55-1.53)$ & 0.261 & -0.328 & 0.743 \\
ASA & 0.834 & $2.30(1.00-5.29)$ & 0.424 & 1.966 & 0.049 \\
Setting & & & & NA & NA \\
Acute & NA & $1.00($ reference) & NA & -1.672 & 0.095 \\
Elective & -1.38 & $0.25(0.05-1.27)$ & 0.825 & -2.22 & 0.026 \\
Screening & -2.382 & $0.09(0.01-0.76)$ & 1.073 & & \\
Location of primary tumor & & & & NA & NA \\
Colon & NA & $1.00($ reference) & NA & 1.335 & 0.182 \\
Rectum & 0.693 & $2.00(0.72-5.53)$ & 0.519 & 0.62 & 0.536 \\
Neo-adjuvant therapy & 0.415 & $1.51(0.41-5.62)$ & 0.669 & 0.038 & 0.970 \\
Tumor stage & 0.008 & $1.01(0.66-1.55)$ & 0.218 & 0.563 & 0.573 \\
Week of surgery & 0.025 & $1.03(0.94-1.12)$ & 0.044 &
\end{tabular}

Table showing results of the multivariate analysis for the risk of patients developing postoperative complications. Abbreviations: ASA American Society of Anesthesiologist, BMI body mass index, $C I$ confidence interval, COVID-19 Coronavirus disease 2019, NA not applicable, OR odds ratio

\section{Declarations}

Conflict of interest The authors declare no competing interests.

Open Access This article is licensed under a Creative Commons Attribution 4.0 International License, which permits use, sharing, adaptation, distribution and reproduction in any medium or format, as long as you give appropriate credit to the original author(s) and the source, provide a link to the Creative Commons licence, and indicate if changes were made. The images or other third party material in this article are included in the article's Creative Commons licence, unless indicated otherwise in a credit line to the material. If material is not included in the article's Creative Commons licence and your intended use is not permitted by statutory regulation or exceeds the permitted use, you will need to obtain permission directly from the copyright holder. To view a copy of this licence, visit http://creativecommons.org/licenses/by/4.0/.

\section{References}

1. Dinmohamed AG, Visser O, Verhoeven RHA, Louwman MWJ, van Nederveen FH, Willems SM, et al. Fewer cancer diagnoses during the COVID-19 epidemic in the Netherlands. Lancet Oncol. 2020 Apr;

2. Health NI for P, and the Environment Ministry of Health W and S. Monitoring and Evaluation of the Colorectal Cancer Screening Programme 2018. 2018.

3. Bronzwaer MES, Depla ACTM, van Lelyveld N, Spanier BWM, Oosterhout YH, van Leerdam ME, et al. Quality assurance of colonoscopy within the Dutch national colorectal cancer screening program. Gastrointest Endosc. 2019 Jan;89(1):1-13.

Publisher's Note Springer Nature remains neutral with regard to jurisdictional claims in published maps and institutional affiliations. 